\title{
Simulación como parte de la Gestión de Crisis
}

Bruno Arias Bailly

\section{RESUMEN}

Éste articulo parte de la problemática que tienen las organizaciones para enfrentarse a eventos inesperados que nunca experimentaron, y de conocer cuán grave podría se para la continuidad del negocio. Así mismo busca la relación que existe entre tres conceptos: La Gestión de Crisis, Simulación de vida y la Gestión del Conocimiento. Por tales razones propone el experimento de colocar a un grupo de miembros de cada organización dentro de un escenario que simule a una empresa ficticia afectada por crisis, para luego mostrar aceptación o rechazo de la simulación como herramienta para la Gestión de Crisis, así como herramienta para crear y compartir conocimiento de forma dinámica. Con el fin de apoyar la misión, se presentan algunas teorías y afirmaciones de diferentes autores relacionadas a conceptos tales como las formas de aprendizaje en el ser humano, crisis, gestión de crisis, simulación, gestión de conocimiento y el modelo de aceptación de tecnologías denominado TAM III. El experimento se llevó a cabo en dos bancos de la ciudad de Lima, los cuales también fueron sometidos a entrevistas y cuestionario que permitieran conocer la percepción de utilidad y fácil uso de la tecnología.

Palabra claves: simulación; continuidad de negocios; gestión de crisis; crisis; modelo de aceptación de tecnologías.

SIMULATION AS A COMPONENT OF CRISIS MANAGEMENT

\section{ABSTRACT}

The principles of this paper are about the problematic that many organizations have, in order to face unexpected events that were never experimented. Furthermore, how severe those events could be for the business continuity. Thus, this study searches for the relation between three concepts: Crisis Management, Live Simulation and Knowledge Management. For those reasons, it purposes to set a group of members from each organization into an experimental scenario that simulates an enterprise in crisis. The idea after the experiment is to accept or reject the usefulness of the simulation as a tool for Crisis Management, likewise, as a dynamic way to share and create knowledge. In order to firm the mission, several theories and assertions are presented -from different authors- in relation to concepts such as, human apprenticeship, crisis, crisis management, simulation, knowledge management and the technology acceptance model TAMIII. The experiment took place in two banks in Lima city. which were exposed to interviews and a questionnaire with the intention to obtain the perception to usefulness and easy of use related to technology.

Keywords: simulation; business continuity; crisis management; crisis; techonology acceptance model.

\section{INTRODUCCION}

La Simulación según su origen etimológico deriva del latín "similis", que puede traducirse como "parecido o similar" y el sufijo "-ion" que es equivalente a "acción o efecto". Por lo tanto se puede decir que simulación es la acción de imitar de algo real, de algún estado o proceso; es por ello que ésta ha sido utilizada para optimizar funcionamientos o rendimientos, seguridad, pruebas, entrenamiento, aprendizaje y educación. Desde hace varios años se han utilizado dispositivos, de acuerdo a la disponibilidad tecnológica de la época, para desarrollar máquinas que permitan simular situaciones reales, habiendo sido desarrollados artificios mecánicos y electrónicos, así como escenarios virtuales, todos estos con el fin de entrenar a individuos en sus diversos campos o hasta prepararse para eventos inesperados, y se usan temas tan variados que pueden ir desde la medicina hasta la utilización en áreas militares.

Éste estudio pretende utilizar como base la necesidad de las empresas de entrenarse en la gestión de crisis a través de algun método o modelo que les permita poner en práctica su plan de continuidad o sus capacidades para tomar decisiones en momentos de crisis, tal y como lo hacen las fuerzas armas con sus juegos de guerra. La teoría del aprendizaje basado en experiencia es un punto importante relacionado a ésta investigación, Rogers (1969) sostiene que las personas aprenden mejor cuando están totalmente involucradas en el proceso de aprendizaje, así como cuando tienen control sobre su naturaleza y dirección. Apoyando a la teoría de Rogers, Heuvel (2012) afirma que el entrenamiento en simulación implica la aceleración de experiencia profesional mediante la confrontación con los desafíos específicos de domino. Así mismo, la gestión de crisis estrategica requiere un conjunto de habilidades "no técnicas" que son diferentes a aquellas aplicadas durante el negocio usual, y que esas habilidades son más facilmente aprendidas, retenidas y transferidas a la vida real a través del entrenamiento frecuente usando ejercicios inmersivos de simulación (Heuvel, 2012). También se toma como apoyo la opión experta del Vice Presidente del área de ingeniería en simulación maritima de la empresa alemana Rheinmetall Defence, C. Grube (comunicación personal, Enero $2,2015)$ confirma que las empresas alemanas usan simulación para la gestión de crisis, especialmente para entrenamiento y análisis.

\footnotetext{
1 Magister en Ingeniería de Sistema e Informática con Mención en GTIC,

Consultor en Simulación y TI en Simulation and Technological System Solutions \& Consulting - SIMTSYS
}

bruno.arias@simtsys.com 
El problema que tiene cualquier empresa es ¿Cómo enfrentarse a eventos inesperados que nunca experimentaron? ¿Cómo saber en que magnitud afectan a la continuidad del negocio o más aún, puede paralizarla?

\section{METODOLOGÍA}

Éste estudio se diseña basado en una estrategia técnico metodologica cualitativa de tipo investigación-acción, que por su objetivo es explicativa ya que pretende resolver por qué no se utiliza Simulación tipo seminario en la Gestión de Crisis, así como qué tan aceptable es el país. La frontera de la investigación esta dada por un escenario ficticio en el que se tome un grupo de colaboradores de por lo menos dos organizaciones (miembros del comité de crisis), en donde se le compartirá información teórica sufiente para aprender a realizar de la mejor forma el manejo de crisis usando simulación tipo seminario. El concepto principal que forma parte de esta investigación es la "simulación tipo seminario" que será medida a través del modelo TAM III en relación a la utilidad y facil uso de la misma, esto permitirá conocer el nivel de percepción al final del experimento. Se planea realizar el experimento dentro de por lo menos dos bancos. Para lograr esto, es necesario contar con un experto en diseño de escenarios para simulación tipo seminario y que a la vez tenga experiencia como evaluador. Al inicio del estudio se proporcionará a cada grupo una serie de preguntas abiertas o entrevistas que reflejen el nivel de conocimiento que los colaboradores tienen acerca de este tipo de simulación y cuánto creen que les puede servir para su desempeño en la toma de decisiones durante el manejo de crisis. Al termino del estudio, se brindará un cuestionario a los participantes, que refleje el nivel de satisfacción que tienen después de la experiencia y cuánto creen en ese momento que la simulación tipo seminario les sirve para su desempeño dentro del manejo de crisis. Con esto se pretende resolver dos interrogantes de investigación: ¿Por qué no se está utilizando simulación de vida en la gestión de crisis (continuidad de negocio) en las organizaciones de nuestro país a pesar que es una herramienta muy importante para éste tipo de gestión en otras parte del mundo? ¿Qué tan aceptable es esa tecnología en nuestro país?

La población requerida debe estar compuesta por el comité de manejo de crisis o por aquellas personas nombradas por la empresa para entrenarse el manejo de crisis, el estudio se llevará a cabo en por lo menos dos bancos de la ciudad de Lima. Los datos serán recopilados de la siguiente manera: 1) Entrevista personal que permitan determinar el nivel de percepción de uso de simulación tipo seminario en la organización, qué tan relacionado o cercano es el concepto de las empresas con respecto a las definiciones teóricas, 2) Desarrollar un escenario fictisio que pueda ser utilizado en un escenario de simulación tipo seminario, con el fin de proporcionar a la empresa la experiencia de usar simulación como herramienta en la gestión de crisis y 3) Analizar las opiniones de los participantes después del experimento, así como la percepción que tiene con relación a su actual entrenamiento en gestión de crisis.

\section{REVISIÓN DE LA LECTURA}

En primer lugar se muestran algunas teorías de aprendizaje en el ser humano, necesarias para entender este fenómeno que es la raíz del caso de estudio pero haciendo énfasis en aquella teoría que se acerca más a fundamentar el objetivo de la investigación. Otros conceptos descritos son la Gestión de Crisis que engloba la propuesta de investigación, el modelo TAM o Technology Acceptance Model por sus siglas en ingles que será tomado por herramienta para medir la aceptación del modelo tecnológico y brevemente la Gestión del Conocimiento. Por último y no por eso menos importante, conoceremos en qué consiste el aprendizaje basado en experiencia a través de la simulación tipo seminario (simulación de vida).

\subsection{El aprendizaje}

Mahar y Harford (2005) citan en su investigación acerca del comportamiento humano, que el producto de aprendizaje incluye conocimiento y habilidades intelectuales, actitudes y respuestas emocionales, así como comportamiento social y habilidades de movimiento. Mahar y Harford encuentran que además, a través de nuestras interacciones con un único ambiente personal, nosotros aprendemos a pensar, actuar y sentir en formas que contribuyen a nuestra identidad humana individual (Mahar y Harford, 2005). Atkins (1994) describe el aprendizaje como una ocurrencia más eficiente cuando: "...todo el cerebro se dedica a pensar, y en particular cuando el proceso de aprendizaje se mueve desde experiencias de reflexión sobre experiencias, así ese patrón o marco de trabajo permite al aprendizaje, la comprensión del significado de aprendizaje en el centro de la mente y finalmente el aprendizaje se mueve sobre una facilidad para usar el lenguaje, reglas, leyes, principios de precisión y eficiencia en el pensamiento; haciéndolo y además aprendiendo" (p.19). 


\subsection{Teoría del aprendizaje basado en experiencia}

Es de hecho ésta teoría la que tiene mayor relevancia para ésta investigación. Rogers (1969), sostiene que el aprendizaje basado en experiencia es auto iniciado, es decir, las personas tienen una inclinación natural para aprender, lo hacen cuando el alumno está totalmente involucrado en el proceso de aprendizaje. Rogers también afirma que el aprendizaje significativo se produce en un entorno donde las amenazas para el aprendiz se reducen al mínimo (Rogers, 1969). Otra afirmación sumamente importante para ésta investigación es aquella Rogers aboga por una situación dinámica en la que el nuevo aprendizaje alimenta e influencia ambientes de aprendizaje existentes, creando así un proceso de cambio continuo (Rogers, 1969).

\subsection{La crisis}

Adawi (2010) define crisis como un evento que se presenta en forma inesperada, el cual afecta las principales actividades de una empresa, dificultando el logro o mantenimiento de los objetivos trazados por la misma. Alcat (2009) manifiesta: me siento convencido de que las empresas que estén preparadas y cuyos directivos invierten en formación y comunicación de crisis, serán capaces de afrontar cualquier contingencia mucho mejor que las que no lo están, y desgraciadamente la mayoría de nuestras empresas no sólo no están preparadas sino que siguen pensando que las crisis les ocurren a otras y nunca a ellas o en el mejor de los casos, que cuando tengan una contingencia grave ya verán en ese momento lo que deben hacer; gravísimo error como punto de partida. Wigodski (2007) presenta la siguiente clasificación de escenarios de crisis: a) Producido por ataques de agentes externos; compuesto por empresas de la competencia, rumores mal intencionados, delincuencia, ataques informáticos, contaminación de productos, terrorismo o guerra con otro Estado; b) Producido por ataques de agentes internos; compuesto por sabotaje o boicot de algún miembro de la empresa por venganza, paralización de labores por huelga de operaciones, accidentes, etc.; c) Producido por accidentes a causa de fenómenos naturales; causados por terremoto, tsunami, inundación por lluvias, entre otros; d) Producido por incidentes involuntarios; incendios, error técnico, falla o demora en la ejecución de algún proceso de la empresa. Adawi afirma que existen empresas -sobre todo en países desarrollados- que cuentan con comités de crisis, que se encargan crear condiciones adversas que paralicen la actividad de la empresa; pero esas condiciones no nacen de la mente de los miembros del comité y tampoco son directamente implementadas para probar el proceso real por el riesgo que implicaría, sino que les sirven para analizar las condiciones o situaciones en las que la empresa sería gravemente afectada en su productividad y luego determinan qué es lo que se debería hacer para superar el evento que generó la crisis (Adawi, 2010).

\subsection{La gestión de crisis}

Álvares y Macías (2007) afirman que la gestión de crisis es un planteamiento basado en el desarrollo de planes de contingencia en los que la respuesta es inmediata y automática de acuerdo a unas capacidades propias y existentes en el momento de la crisis y que se activan mendiante unos procedimientos preestablecidos. Por otro lado, la gestión de crisis consiste en contar con la capacidad para reconocer y actuar frente a las señales que anticipan una situación perjudicial para la empresa (Wigodski, 2007). Heuvel (2012) sostiene que cualquier gestión de crisis debe fundamentalmente ser soportada por una estructura predefinida y un arreglo de planes que permitan asegurar una recuperación y respuesta apropiadas ante un incidente amenzante.

\subsection{Modelo TAM}

En su modelo inicial Davis (1985) sugieren que la motivación del usuario se explica por tres factores: Fácil uso percibido, utilidad percibida y la actitud hacia el uso del sistema. Lee, Kozar y Larsen (2003) afirman que TAM se ha vuelto tan popular que ha sido citado en la mayor parte de investigaciones que se han ocupado de la aceptación del usuario por tecnología. La utilidad percibida y la percepción de facilidad de uso de la siguiente manera: la utilidad percibida como el grado que una persona cree, que el uso de un sistema en particular podría mejorar su desempeño en el trabajo; el fácil uso percibido como el grado que un individuo cree, que el uso de un sistema en particular sería de libre de esfuerzo físico y mental (Davis, 1985). Con el fin de desarrollar unas escalas de medición de la utilidad percibida y la percepción de facilidad de uso, Davis refiere a las escalas psicométricas utilizadas por la psicología, las mismas que típicamente incitan al individuo a responder diversas preguntas que se refieren a un contexto dado (Davis, 1989). Las respuestas obtenidas de estas indicaciones se pueden analizar, y se utilizan como un indicador de la creencia interna de una persona para el contexto considerado. En el caso de TAM, Davis desarrolló sus escalas psicométricas tanto para la percepción de utilidad y para la percepción de fácil uso, en tres fases: una fase de pruebas preliminares, y estudio de campo empírico, y un experimento de laboratorio (Davis, 1989). Lee, Kozar, y Larsen (2003) afirman 
que el modelo de aceptación de tecnología (TAM) se considera la teoría más influyente y comúnmente empleada para la descripción y la aceptación de los sistemas de información por el individuo.

\subsection{Gestión del conocimiento}

La gestión del conocimiento es en esencia la actividad que permite facilitar los procesos mediante los cuales las organizaciones y las personas crean, comparten y utilizan el conocimiento. Shannak, Masa'deh y Akour (2013) explican que las estrategias para la gestión del conocimiento deben estar alineadas con la visión y la gestión, además deben asegurar que los empleados de la organización estén embarcados en las estrategias. Así mismo, Shannak, et. al (2013) sostienen que debe existir retro-alimentación entre los ejecutivos y los empleados de la organización. Si bien es cierto que la gestión del conocimiento sortea una amplio rango de disciplinas y tecnologías tales como sistemas expertos, groupware, redes semánticas, entre otros, una tecnología en particularmente es la que centra mayor atención para ésta investigación y es la simulación, que el experto en Gestión del Conocimiento Karl-Erik Sveiby Profesor de Gestión del Conocimiento de la Escuela de Negocios Hanken en Helsinki, Finlandia, sugiere como un componente tecnológico de gestión de conocimiento, refiriéndose a "la simulación por computadores", simulación manual como juegos de rol para probar las habilidades de los jugadores.

\subsection{Aprendizaje virtual}

El aprendizaje virtual tiene como herramienta a la simulación, la cual está compuesta por la simulación virtual, constructiva, constructiva-distribuida y de vida o tipo seminario. Apoyando a la teoría de Rogers del aprendizaje basado en experiencia, Heuvel (2012) sostiene partiendo de la premisa de que las habilidades complejas se adquieren mejor en base a la experiencia, el entrenamiento en simulación implica la aceleración de experiencia profesional mediante la confrontación con los desafíos específicos de domino. Schank (2001) afirma que los puntos principales del aprendizaje virtual son: La gente necesita estar motivada para aprender; la gente tiene que experimentar sus expectativas de fallar para aprender; el aprendizaje debe ser divertido; y el aprendizaje debe basarse en una historia. Por otro lado, Dawley y Dede (2013) afirman que el aprendizaje se sitúa en el momento que un estudiante experimenta y aplica el aprendizaje en un entorno específico o situaciones que tienen sus propios contextos sociales, físicos y culturales.

Según Gibson (2010) las tecnologías virtuales proveen ambientes alternativos de aprendizaje, porque disponen o pueden ser creados una variedad casi infinita de contextos virtuales, lo que les da a los usuarios una sensación de "estar inmerso en la situación" y por lo tanto, la capacidad de aplicar el aprendizaje en un contexto real. Heuvel (2012) afirma que la alta fidelidad y los ejercicios inmersos en simulación (haciendo referencia a la simulación de vida) sin duda son la forma más apropiada de formación para ejecutivos de nivel estratégico en la gestión de crisis. Esto debido a que proporciona a los ejecutivos la oportunidad de practicar sus respuestas a crisis en condiciones semejantes de estrés, incertidumbre, dinamicidad de riesgo y rendición de cuentas como la experimentada en incidentes de bienes.

La exposición a incidentes simulados dentro de un ambiente de aprendizaje seguro permite la adquisición y desarrollo de habilidades críticas y transferibles que son cruciales para la gestión de crisis eficaz dentro de cualquier dominio u organización (Heuvel, 2012).

\section{8 ¿Como organizar los ejercicios de simula- ción para la gestión de crisis?}

Adawi (2010) sostiene que todo ejercicio de simulación debe contar con diferentes grupos o equipos organizados, por un lado aquel que diseñe, conduzca, controle y evalué el ejercicio y por otro lado aquel compuesto por los participantes que serán los que se entrenen en la gestión exitosa de la crisis. Según Adawi, el grupo de dirección del juego debe estar conformado por un gerente de mando superior, de ser posible el Gerente General o algún miembro del Directorio de la empresa, conjuntamente con un grupo de gerentes o ejecutivos que cuenten con las especialidades necesarias para poder determinar y diseñar el escenario ideal para los objetivos que se persigan en el juego (Adawi, 2010). Adawi también explica que el grupo de conducción y evaluación del juego será responsable de la ejecución, conducción del juego, implementación de las entradas laterales, personificación de los roles, tales como medios de prensa u otras organizaciones que no estén siendo representadas por el grupo de participantes que formen parte del entrenamiento (Adawi, 2010).

Adawi explica que para la función de evaluación es deseable que se cuente con expertos en los procesos y áreas que se están afectando con la crisis quienes deberán designar como mínimo un facilitador por cada grupo de participantes del entrenamiento, cuya función es de apoyo y guía para el entrenamiento del escenario y entradas laterales así como,e I grupo de participantes los constituyen los ejecutivos que se están entrenando; y que por último el grupo de prensa provee de una ambiente de mayor realismo en el juego (Adawi, 2010). 
Gibson (2010) afirma que para crear un mundo virtual se deben generar estímulos sensoriales tales como gráficos, sonidos, percepciones visuales de movimiento a través del medio ambiente, la capacidad de tocar objetos y mapas que proporcionen la ubicación geográfica, lo cual crea la sensación de inmersión del ser humano dentro de un ambiente simulado. Gibson explica que la idea en estos mundos virtuales es enseñar a los aprendices una serie de actividades en forma interactiva y basada en experiencia que les permita tomar decisiones y obtener diferentes resultados de acuerdo a las acciones tomadas (Gibson, 2010).

\section{PRESENTACIÓN Y ANALISIS DE DATOS}

Tiene como fin mostrar inicialmente el nivel de concepción que tienen las entidades bancarias en la relación a crisis, riesgos, entrenamiento, simulación tipo seminario, entre otros. En segundo lugar mostrar los resultados de los cuestionarios aplicados al término de los experimentos que miden la percepción de los participantes en relación a la utilidad, fácil uso, entre otros constructos que el modelo TAM III proporciona. Es así que se obtiene la aceptación del modelo o tecnología que se evalúa.

\subsection{Análisis}

Durante en la primera fase, se elaboró un conjunto de 12 preguntas relacionadas a conceptos tales crisis, riesgo, planeamiento, simulación, gestión de conocimiento y entrenamiento. Se invitó a 14 de las 16 bancos en Lima para que designe a por lo menos a un miembro del comité de crisis para ser entrevistado, sólo 2 bancos estuvieron dispuestos a colaborar con la investigación. Las entrevistas fueron grabadas y se llevaron a cabo dentro de las instalaciones de ambos bancos. Al término de cada entrevista se procedió a realizar la transcripción de las mismas dentro del software Atlas.ti, con el fin de codificar los constructos basados en las citas de interés para ésta investigación. Para la implementación del juego de simulación, fue necesario elaborar un escenario ficticio de una entidad bancaria que muestra cómo ésta opera en relación al servicio que brinda y cómo está constituida organizacional y geográficamente. En la tercera fase se desarroIló un cuestionario de 43 preguntas utilizando una variante de Likert, compuesta por un formulario en formato .PDF y que se envió al correo electrónico de cada uno de los participantes del juego de simulación de cada banco, esto con el fin de conocer la percepción que tienen con respecto a la utilidad, fácil uso e intensión de usar la tecnología propuesta. Las preguntas del cuestionario estaban agrupadas de acuerdo a los constructos que presenta el modelo TAM y fueron validadas de acuerdo al juicio de 2 expertos en simulación en gestión de crisis y juegos de guerra, uno de ellos acompaño el desarrollo de ésta investigación.

\subsection{Presentación de resultados}

Los bancos participantes denominan "Banco 1" y "Banco 2". Los resultados obtenidos de las entrevistas iniciales con relación a la codificación realizada en el software ATLAS.ti permiten conocer cómo relacionan cada uno de los constructos. La red semántica muestra la relación entre los códigos, las citas que presenta cada código y sí tienen citas en común tal y como se muestra en la figura 1.

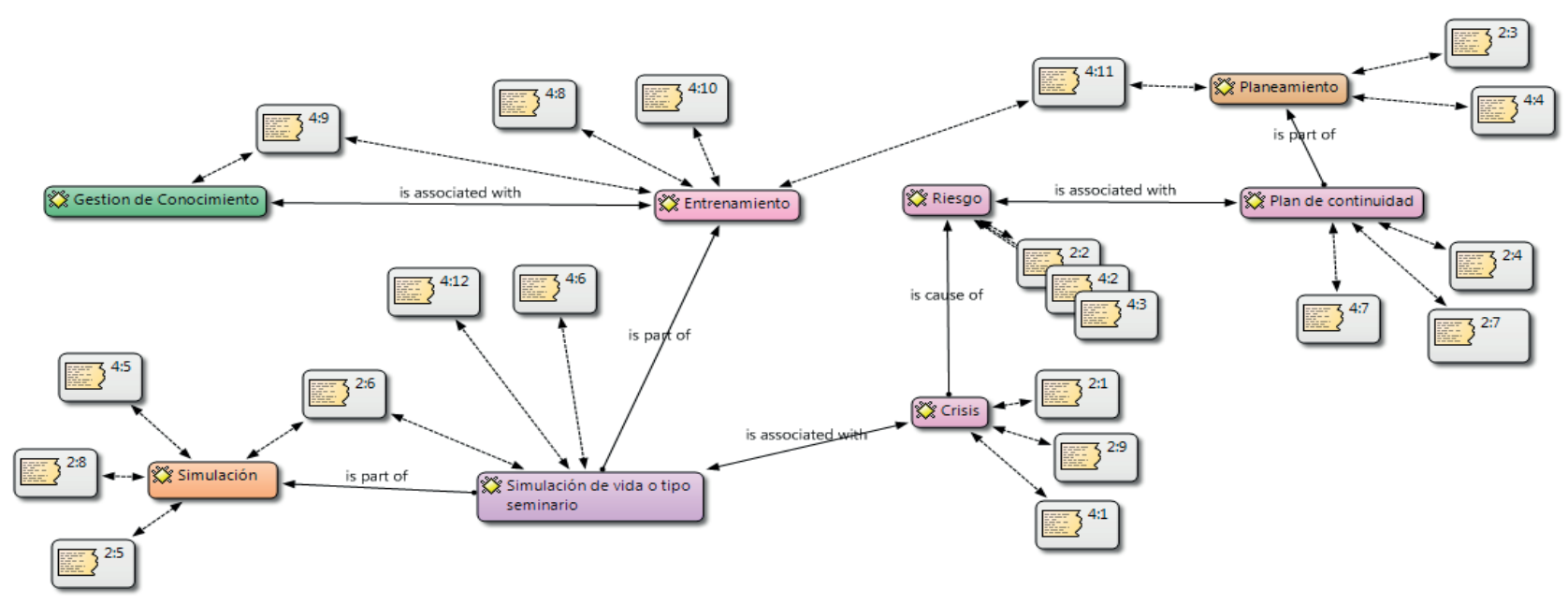

Figura 1: Red semántica. Fuente: Elaboración Propia. 
De la red semántica se interpreta que para los entrevistados en ambos bancos la simulación de vida o tipo seminario, es parte de la simulación, así como parte del entrenamiento. También coinciden que el entrenamiento está asociado a la gestión del conocimiento. Por otro lado, se entiende que el plan de continuidad es parte del planeamiento y que está directamente asociando con los riesgos; los mismos que pueden causar una crisis. Así mismo perciben que la simulación tipo seminario está asociada a la crisis, sin embargo no han tenido la oportunidad de poner el modelo a prueba. La simulación tipo seminario es parte de la simulación y que a su vez es parte del entrenamiento, que su vez está relacionado con la gestión del conocimiento. El plan de continuidad es parte del planeamiento que realiza un banco y guarda una asociación directa con los riesgos, los cuales pueden ser la causa de crisis en una organización. Por último, la simulación tipo seminario, está asociada a la crisis, en sentido que permite entrenarse para evitarla o tomar las mejores decisiones. La segunda etapa corresponde al juego de simulación y al análisis del cuestionario. Los valores de las respuestas por cada participante fueron agrupados por cada constructo del modelo TAM e ingresados al software SPSS, en donde se crearon variables para obtener la Media de cada uno de los constructos. Estos criterios se toman como referencia para ver la aprobación porcentual de cada constructo en la gráfica del modelo TAM tal y como se muestra en la figura 2 , con el fin de analizar la relación de aceptación, es así que si los constructos de "calidad de la información", "influencia social" y "expectativa de rendimiento" son aceptados, entonces incrementa la evidencia en relación a la utilidad percibida. Además, si el constructo de "utilidad percibida" es aceptado, entonces también incrementa la evidencia en relación a la intención de usar el sistema o modelo. A través de este modelo se puede contar con la capacidad de concluir sí la tecnología es aceptada por los usuarios.

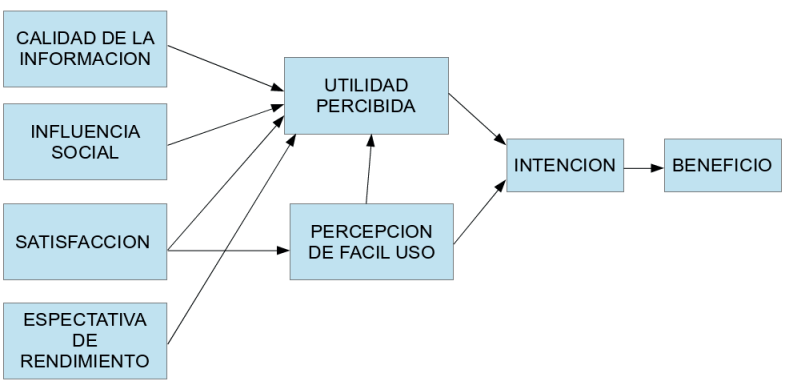

Figura 2: Modelo TAM III. Fuente: Elaboración Propia.

\subsection{Resultados del cuestionario en el Banco 1}

En respuesta al cuestionario, se aceptaron 6 de los 7 constructos propuestos. El constructo rechazado fue el de intensión de uso. Las figuras 3 y 4 a continuación muestran dos gráficas obtenidas del Banco 1 relacionadas a los constructos de: 1) percepción de utilidad, en donde se puede observar que el $80 \%$ de los participantes considera a la simulación tipo seminario muy útil para desarrollar sus labores, mientras que un $20 \%$ la considera totalmente útil; y de 2)Intensión de uso, en donde ser observa que un $20 \%$ de los participantes considera muy desfavorable su intención de usar simulación próximamente, un $40 \%$ considera desfavorable y un $40 \%$ considera favorable su intensión de uso.
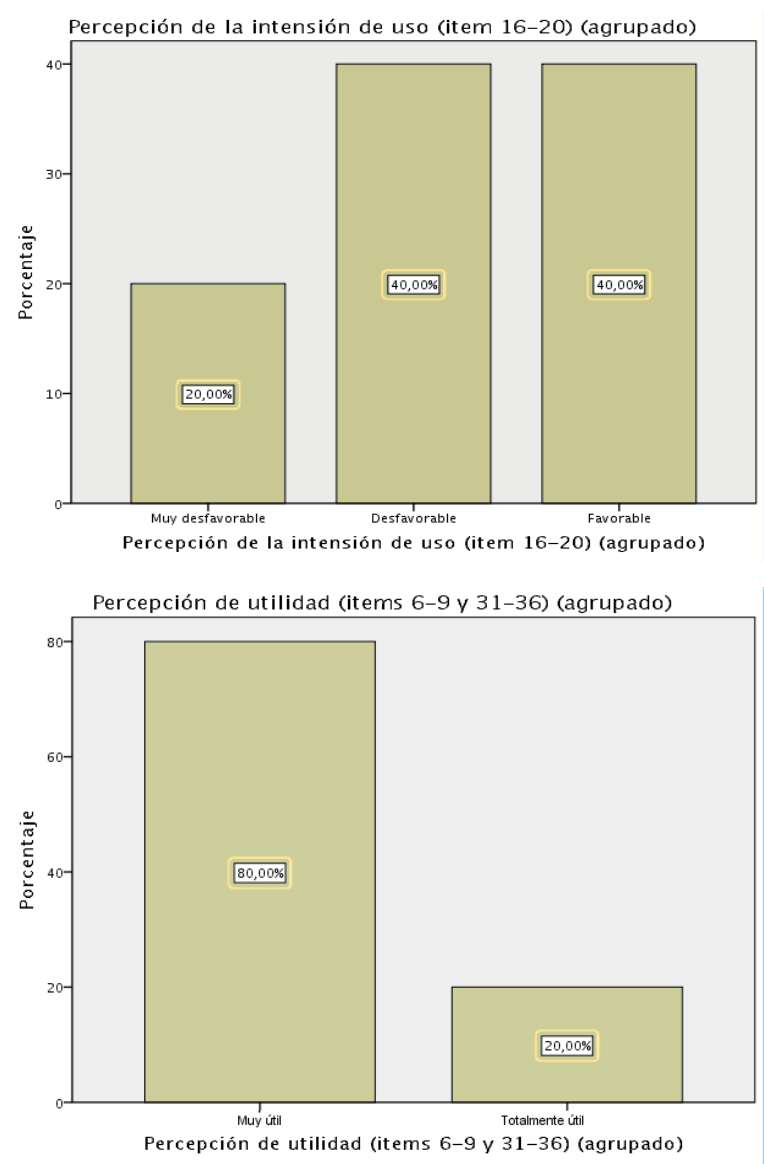

Figura 3: Utilidad percibida

Figura4: Intensión de uso

Fuente: Elaboración propia.

\subsection{Resultados del cuestionario en el Banco 2}

En respuesta al cuestionario, se aceptaron todos constructos propuestos. Las figuras 5 y 6 a continuación muestran dos gráficas obtenidas del Banco 2 relacionadas a los constructos de: 1) percepción de utilidad, en donde se puede observar que el 
$77,78 \%$ de los participantes percibe a la simulación tipo seminario muy útil para desarrollar sus labores, mientras que un $22,22 \%$ la percibe totalmente útil; y de 2)Intensión de uso, en donde ser observa que un $66,67 \%$ de los participantes considera favorable su intención de usar simulación próximamente, un $33,33 \%$ considera muy favorable su intensión de uso.
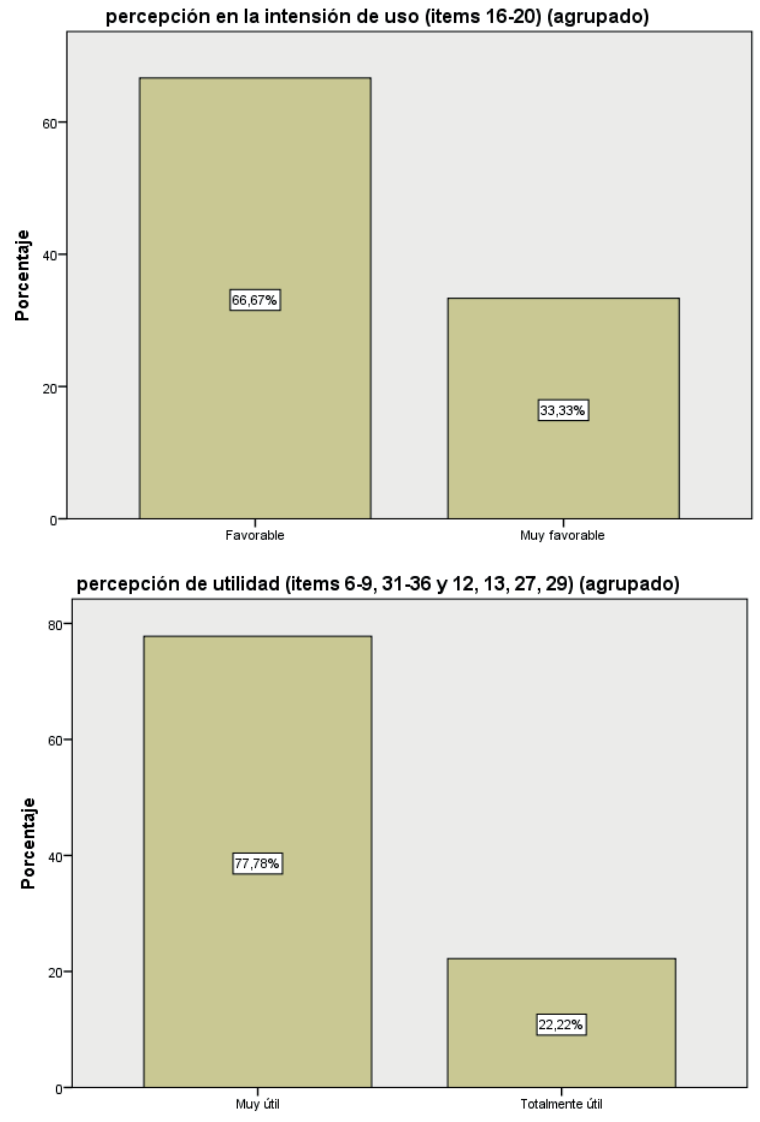

Figura 5: Utilidad percibida.

Figura 6: Intensión de uso

Fuente: Elaboración propia.

\section{CONCLUSIONES}

Respondiendo a la primera pregunta de investigación de ¿Por qué no se está utilizando simulación de vida en la gestión de crisis (continuidad de negocio) en las organizaciones de nuestro país a pesar que es una herramienta muy importante para éste tipo de gestión en otras parte del mundo? Las entrevistas y la red semántica reflejan que tienen un conocimiento claro de la importancia del entrenamiento en la gestión de crisis y que de hecho se entrenan con modelos similares pero no tan dinámicos, así mismo, perciben que la simulación está relacionada al entrenamiento. Sin embargo, no contaban con experiencia en este tipo de tecnología, así como, tampoco tenían conocimiento que otros países alrededor del mundo la usan no sólo en el ámbito financiero. En relación a la segunda y última pregunta de investigación ¿Qué tan aceptable es esa tecnología en nuestro país? En el caso de Banco 1, los resultados del cuestionario aplicado arrojaron que 6 de los 7 constructos fueron aceptados, por lo tanto se puede concluir que la simulación tipo seminario es aceptada en un $86 \%$ como método de entrenamiento en la gestión de crisis en esa entidad bancaria. Para el caso del Banco 2, los resultados del cuestionario aplicado arrojó la aceptación de los siete constructos. Por lo tanto, siguiendo la relación de constructos del modelo TAM III, y basado en los resultados que arrojan el $100 \%$ de aceptación de los constructos para el caso del Banco 2, se puede concluir que la simulación tipo seminario es totalmente aceptada. Adicionalmente se puede concluir que debido a que ambas entidades bancarias consideran a la simulación tipo seminario como una herramienta útil y fácil de usa para entrenarse en la gestión de crisis, entonces ésta tecnología es aceptada para cumplir con la mencionada labor.

Si bien esta investigación por su metodología buscaba medir la opinión de utilidad y fácil uso de simulación (como modelo) particularmente para la gestión de crisis a través de un trabajo más de escritorio y apoyado por algunas herramientas visuales tales como Powerpoint, lo ideal es apoyar los juegos de simulación con un software simulador que permita a los participantes estar más inmersos en la realidad virtual. Esto último deja dos posibilidades de investigación; la primera (cómo continuación de esta investigación) podría ser medir a la simulación dentro de otras entidades tales como el gobierno o mineras; y la segunda como parte de una investigación tecnológica que permita construir una herramienta de simulación utilizando inteligencia artificial, motores gráficos y otras áreas de la informática, aprovechando que las tecnologías de información son cada vez más potentes.

\section{REFERENCIAS BIBLIOGRÁFICAS}

[1] Adawi, L. (2010). Simulación para el entrenamiento en gestión de crisis. Revista de Marina, 3, 35-45.

[2] Alcat, E. (2008). ¿Crisis? Si, gracias. La Comunicación Minimiza el Impacto de una Crisis. Revista de Comunicación, 7, 28-32. Recuperado de http://pdfs.wke.es/2/9/1/3/ pd0000022913.pdf

[3] Álvares, C., Macías, J. (Ed.). (2007), Manual de procedimiento en gestión de crisis, Madrid: Sociedad Española de Medicina de Catástrofes. 
[4] Atkin, J. (1999). Thinking: critical for learning, Edición avanzada en línea. Recuperado de http://learning-by-design.com/papers/ thinking crit for Ing.pdf

[5] Davis, F. (1985). A technology acceptance model for empirically testing new end-user information systems: theory and results (Unpublished Doctoral dissertation), MIT Sloan School of Management, Cambridge, MA.

[6] Davis, F. (1989). Perceived usefulness, perceive easy to use, and user acceptance of information technology. MIS Quaterly, 13, 319340.

[7] Dawley, L., Dede, C. (2013). Situated Learning in Virtual Worlds and Immersive Simulations. USA: Springer.

[8] Gibson, D. (2010). Living virtually: Researching new worlds. USA: Peter Lang Publishing

[9] Heuvel, C. (2012), High Fidelity Simulation Exercises for Training Strategic Crisis Management. The Business Continuity and Resiliency Journal, 2, 4-14.

[10] Lee, Y., Kozar, K. A., \& Larsen, K. R. T. (2003), The Technology Acceptance Model: Past, Present, and Future, Communication for the Association for Information Systems, 12, 752780.
[11] Mahar, S., Harford, M. (2005). Research on Human Learning. Department of Education and Training. (1). Recuperado de: https://www. researchgate.net/publication/265173642 Research_on_Human_Learning Background Paper

[12] Rogers, C. (1969). Freedom to learn: a view of what education might become. USA: C. E. Merrill

[13] Schank, R. (2001). Virtual Learning A Revolutionary Approach to Building a Highly Skilled Workforce. International Society for Performance Improvement, 5, 39-41. doi: 10.1002/pfi.4140400511

[14] Shannak, R. O., Masa'deh, R. M., Akour, M. A. (2013). Knowledge Management Strategy Building. European Scientific Journal. 8(15). Recuperado de: http://eujournal.org/index. php/esj/article/view/236/1543

[15] Wigodski, T. (2007), Gestión de Crisis: Nuevas Capacidades para un Mundo Complejo [Documento de Trabajo], Universidad de Chile, 93, 1-13. 